\title{
Diferencias en la ansiedad y depresión por variables contextuales en mujeres con cáncer de mama
}

\author{
Differences in Anxiety and Depression by Contextual \\ Variables in Women with Breast Cancer
}

\author{
Eva Magdalena García-Hernández ${ }^{1}$ \\ (D) https://orcid.org/0000-0001-6826-8335 \\ San Juana López-Guevara² \\ https://orcid.org/0000-0002-2984-3576 \\ Luz Elena Cano-Fajardo ${ }^{3}$ \\ D https://orcid.org/0000-0001-9404-3666
}

\author{
Maribel Avila-Medina ${ }^{4}$ \\ (D) https://orcid.org/0000-0003-3094-8831 \\ Tirso Duran-Badillo 5 \\ https://orcid.org/0000-0002-7274-3511 \\ Delia Ponce-Martínez ${ }^{6}$ \\ https://orcid.org/0000-0002-3588-4569
}

1,2,3,4,5,6Unidad Académica Multidisciplinaria Matamoros, Universidad Autónoma de Tamaulipas, México

Resumen. Objetivo. Identificar las diferencias en la ansiedad y depresión de acuerdo con variables contextuales en mujeres con cáncer de mama que se encuentran bajo tratamiento. Método. Estudio comparativo realizado en 91 mujeres con cáncer de mama, en tres unidades de atención del Noreste de México. Se utilizó la Escala Hospitalaria de Ansiedad y Depresión. Resultados. El 51.6\% ( $f=47)$ resultó con ansiedad y el 25.3\% ( $f=23)$ depresión. La ansiedad y depresión no es diferente de acuerdo con el grado de escolaridad $(p>.05)$ y estado civil $(p>.05)$. La depresión $(p=.001)$ y ansiedad $(p=.05)$ es diferente de acuerdo con la unidad de atención. Se concluye que es importante cuidar el estado emocional de las pacientes con cáncer, ya que se observó que la mitad de las pacientes presentaron ansiedad y una cuarta parte depresión. Es necesario analizar el contexto, ya que la depresión y ansiedad son diferentes de acuerdo con la unidad de atención.

Palabras clave. Ansiedad, depresión, neoplasias, cáncer.

Abstract. Objective. The aim is to identify the differences between anxiety and depression according to contextual variables in women with breast cancer who are under treatment. Method. A comparative study was carried out in 91 women with breast cancer in three care units in Northeast Mexico. The Hospital Anxiety and Depression Scale were used. Results. The 51.6\% $(f=47)$ resulted in anxiety and $25.3 \%(f=23)$ depression. Anxiety and depression are not different according to the degree of schooling $(p>.05)$ and marital status $(p>.05)$. Depression $(p=.001)$ and anxiety $(p=05)$ is different according to the unit of attention. In conclusion, it is important to take care of the emotional state of cancer patients since it was observed that half of the patients presented anxiety and a quarter with depression. It is necessary to analyze the context because depression and anxiety are different regarding the unit of attention.

Keywords. Anxiety, Depression, Neoplasm, Cancer.

${ }^{1}$ Eva Magdalena García-Hernández. Universidad Autónoma de Tamaulipas, Unidad Académica Multidisciplinaria Matamoros, México. E-mail: eva7magdalena@hotmail.com

${ }^{2}$ San Juana López-Guevara. Universidad Autónoma de Tamaulipas, Unidad Académica Multidisciplinaria Matamoros, México. E-mail: sjlopez@docentes.uat.edu.mx ${ }^{3}$ Luz Elena Cano-Fajardo.Universidad Autónoma de Tamaulipas, Unidad Académica Multidisciplinaria Matamoros, México. E-mail: Iuzelena_nurse@hotmail.com ${ }^{4}$ Maribel Ávila-Medina. Universidad Autónoma de Tamaulipas, Unidad Académica Multidisciplinaria Matamoros, México. E-mail: mavila@docentes.uat.edu.mx ${ }^{5}$ Tirso Duran-Badillo. Universidad Autónoma de Tamaulipas, Unidad Académica Multidisciplinaria Matamoros. Dirección postal: Av del Maestro y Marte S/N. Colonia Alianza, H. Matamoros, Tamaulipas, México. C.P. 87410. E-mail: tirdb27@hotmail.com

${ }^{6}$ Delia Ponce-Martínez. Universidad Autónoma de Tamaulipas, Unidad Académica Multidisciplinaria Matamoros, México. E-mail: dponce@docentes.uat.edu.mx

\section{(C) $(\mathcal{D} \Theta \Theta$}

Esta obra está bajo una licencia de Creative Commons Reconocimiento-NoComercial-SinObraDerivada 4.0 Internacional. 


\section{Introducción}

El cáncer es uno de los principales problemas de salud pública en el mundo. Ocasiona serios problemas en las diferentes esferas de la vida, incluyendo la cuestión emocional. Por lo que se considera importante la identificación y atención de problemas psicológicos como la ansiedad y depresión en pacientes que presentan estas características.

El cáncer es la causa del 12\% de las defunciones a escala mundial, las predicciones indican que, en los próximos veinte años, el número anual de defunciones por cáncer pasará de 6 millones a 10 millones. Unos de los principales factores que contribuyen a este aumento previsto son la creciente proporción mundial de incidencia de diferentes tipos de cáncer. Como resultado de esto actualmente existen unas 20 millones de personas que lo padecen; y es probable que en el 2020 la cifra supere los 30 millones (Organización Mundial de la Salud [OMS], 2012). El cáncer de mama es uno de los principales problemas de salud pública a nivel mundial, produciendo aproximadamente 571000 defunciones (OMS, 2018a).

En el afrontamiento del diagnóstico de una enfermedad oncológica se presentan respuestas cognitivas y conductuales en los pacientes (Watson et al., 1988). Es decir, ante un evento vital estresante, como el diagnóstico de cáncer, la persona realiza una interpretación y evaluación de la situación. Si la interpreta como una amenaza y carece de recursos para afrontar las consecuencias negativas, se generará una reacción emocional caracterizada por ansiedad. En cambio, si la persona valora la situación como una pérdida importante (de salud, de funcionalidad, de esperanza de vida) generará una reacción emocional de tristeza que puede desencadenar en una depresión (Folkman \& Lazarus, 1984).

Almanza-Muñoz y Holland (2000) plantean que, aunque el diagnóstico de cáncer se ha logrado enfrentar con mayor optimismo, se cursa con ansiedad y depresión; donde el contexto y servicios médicos son importantes para el afrontamiento. En cuanto al contexto, se ha encontrado que el grado de depresión es diferente de acuerdo con el nivel de escolaridad (Berrospi-Reyna et al., 2017); y es similar tanto en los que reportaron trabajar como en aquellos que se encuentran desempleados; y es similar en personas solteras, viudas, casadas o divorciadas (Lerma-Talamantes et al., 2017).

Respecto a la ansiedad, se ha reportado que no existen diferencias por situación laboral y estado civil (Lerma-Talamantes et al., 2017). Tanto para la depresión como para la ansiedad, no se han identificado estudios donde se reporte análisis de diferencias de acuerdo con el conocimiento de la etapa clínica del cáncer y unidad en la que recibe la atención médica.

Esta situación resulta preocupante porque se ha documentado que el cáncer supone un desequilibrio en la vida de las pacientes, ya que no solo afecta el estado físico, sino que también influye en otras áreas como el bienestar emocional, las relaciones sociales, la autoestima y el no poder realizar las actividades como antes. Las pacientes que presentan una mayor alteración de su imagen corporal presentan mayores niveles de ansiedad y depresión, además de una autoestima más baja (Pintado-Cucarella, 2016).

Se entiende por ansiedad a los sentimientos que involucran aspectos fisiológicos y psicológicos, tales como el estado emocional en el que el paciente presenta activación fisiológica; así como elementos cognitivos de aprensión, culpa y sensación de desastre inminente. Se distingue del temor, que es una reacción emocional donde se conoce el objeto causante del sentimiento (Sarason \& Sarason, 2006).

Por otra parte, se ha documentado que la ansiedad es una respuesta ante la percepción y evaluación subjetiva de una situación o acontecimiento amenazante (Sandín \& Chorot, 1995); 
por lo que, como mecanismo de respuesta, el organismo puede prepararse para responder a las situaciones que se presenten en el entorno, o al anticiparse a un peligro irreal.

Otra alteración emocional que se presenta ante el diagnóstico de cáncer es la depresión, la que es considerada una alteración del estado de ánimo que genera pérdida de interés para realizar las actividades cotidianas, pérdida de la confianza en sí mismo, sentimiento de inferioridad, ideas de culpa, falta de concentración, exceso de cansancio e inutilidad (OMS, 2018b). Una de las herramientas más prácticas y económicas para la detección oportuna de sintomatología ansiosa y depresiva la constituyen los instrumentos de tamizaje validados y confiables; ya que proporcionan una aproximación para la detección de posibles casos clínicos (Galindo et al., 2015). Tanto la ansiedad como la depresión en pacientes con cáncer puede ser medida con la Escala de Ansiedad y Depresión Hospitalaria (Zigmond \& Snaith, 1983).

Estudios realizados han demostrado altos porcentajes de depresión y ansiedad. En Colombia se encontró ansiedad clínica en el $84.1 \%$ de las mujeres con cáncer; y depresión clínica en el 25.2\% (Valderrama \& Sánchez, 2017). En un estudio realizado en México, en mujeres con diagnóstico de cáncer se encontró que el 61.3\% manifestaron síntomas depresivos clínicamente significativos; de ellas el 22.1\% presento depresión leve, 23.1\% moderada y el 16.6\% depresión severa (Fuchs-Tarlovsky et al., 2013).

La evidencia científica muestra que en la población oncológica del 15 al 58\% de pacientes presentan sintomatología depresiva, y de un 24 a 66\% sintomatología ansiosa, los cuales representan índices superiores a los reportados entre la población general. La ansiedad y depresión en pacientes oncológicos puede influir negativamente en la calidad de vida, duración del ingreso hospitalario, ideación suicida, autocuidado, adhesión al tratamiento médico y síntomas como dolor, náuseas, vómito y fatiga. Por lo que se considera que es necesaria la identificación y atención de problemas psicológicos en pacientes que presentan estas características (Galindo et al., 2015).

Con base en lo descrito se realizó un estudio con la intención de identificar las diferencias en la ansiedad y depresión de acuerdo con variables contextuales en mujeres con cáncer de mama que se encuentran bajo tratamiento. Se pretende que los resultados del presente estudio incrementen el cuerpo de conocimientos para orientar las intervenciones interdependientes con impacto en la situación emocional de esta población vulnerable, a través de la detección temprana.

\section{Método}

Diseño de estudio y participantes

El estudio fue de tipo descriptivo y comparativo. La población está compuesta por mujeres con diagnóstico de cáncer de mama que son atendidas en tres hospitales (unidades de atención públicas) ubicados en Matamoros Tamaulipas, al noreste de México. En México el sistema de salud se clasifica en público y privado; dentro del público se encuentran el Instituto Mexicano del Seguro Social (IMSS) que atiende a trabajadores asalariados jubilados y sus familias, el Instituto de Seguridad y Servicios Sociales de los Trabajadores del Estado (ISSSTE) que brinda el servicio de salud a los asalariados, jubilados y sus familias, y el Hospital General para atender a autoempleados, trabajadores del sector informal, desempleados y sus familias (Gómez et al., 2011). La población que participó en este estudio correspondió a mujeres que son atendidas en los centros ya mencionados, por tratarse de una población pequeña, se incluyó a las 91 pacientes que se encontraban 
bajo tratamiento oncológico en el momento de la recolección de datos. Se incluyó a mujeres con diagnóstico de cáncer de mama, mayores de 18 años y residentes en Matamoros, Tamaulipas, México.

\section{Instrumentos}

Escala Hospitalaria de Ansiedad y Depresión (Zigmond \& Snaith, 1983). Contiene 14 ítems, siete miden la ansiedad y siete la depresión. La opción de respuesta es en escala tipo Likert que oscilan del cero al tres. Los ítems 1, 3, 5, 7, 9, 11 y 13 corresponden a la subescala de ansiedad, y los ítems 2, 4, 6, 8, 10, 12 y 14 a la subescala de depresión. Para el análisis se hace una sumatoria por subescala, con un puntaje mínimo de cero y máximo de 21. Los puntos de corte de 0 a 7 indican normal, de 8 a 10 sugiere caso probable de ansiedad y/o depresión, y del 11 al 21 caso de ansiedad y/o depresión. Esta escala fue validada en población de pacientes oncológicos mexicanos y presentó una consistencia interna de alpha de Cronbach de .86 para la escala global, .79 para la subescala de ansiedad y .80 para la de depresión (Galindo et al., 2015).

Las variables contextuales fueron medidas a través de una cédula de datos personales, en la que se valoró si la condición laboral (empleado o desempleado), estado civil (soltera, casada, viuda o divorciada), nivel de escolaridad (ninguno, primaria, secundaria, preparatoria o universidad), si tienen conocimiento de la etapa clínica del cáncer y la unidad en que reciben la atención (IMSS, ISSSTE u Hospital General).

\section{Procedimientos}

El estudio se apegó a los principios éticos para la investigación médica en seres humanos plasmados en la Declaración de Helsinki de la Asociación Médica Mundial. De manera inicial se solicitó la autorización del comité de Investigación y Ética de la Unidad Académica Multidisciplinaria Matamoros de la Universidad Autónoma de Tamaulipas, con número de registro 022. En seguida se solicitó la autorización de las autoridades correspondientes para realizar el trabajo de campo. En el primer semestre del 2018 el investigador principal acudió con cada uno de los pacientes en la sala de quimioterapia, una vez iniciado el tratamiento, se les hizo la invitación a participar, se les informó sobre los aspectos relacionado con la investigación, se aclararon dudas; y finalmente se solicitó la firma del consentimiento informado y procedió a la aplicación del instrumento.

\section{Análisis de datos}

Los datos fueron procesados y analizados en el programa estadístico IBM-SPSS versión 21 para Windows, se utilizó estadística descriptiva, en seguida se aplicó la prueba de Kolmogorov Smirnov; y debido a que no se observó distribución normal en las variables, se optó por utilizar las pruebas de Correlación de Spearman, U de Mann Whitney y H de Kruskal Wallis. Se consideraron relación y diferencias estadísticamente significativas para $p \leq .05$.

\section{Resultados}

La edad promedio fue de 50.22 años ( $D T=10.302)$. El 31.9\% $(f=29)$ con estudio de secundaria, el 36.3\% ( $f=33)$ indicaron estar casadas, el 57.1\% $(f=52)$ reportaron estar desempleadas y el $47.3 \%(f=43)$ correspondió a pacientes que son atendidos en un hospital general. El 51.6\% $(f=47)$ resultó con ansiedad y el 25.3\% ( $f=23)$ con depresión (Tabla 1).

No se encontró relación estadísticamente significativa entre la ansiedad ( $r s=-.15 ; p=.15)$ y depresión ( $r s=-.16 ; p=12$ ) con la edad de las pacientes. Se observó que la ansiedad en las 
Tabla 1

Descripción de los pacientes, depresión y ansiedad

\begin{tabular}{|c|c|c|}
\hline Variables & $f$ & $\%$ \\
\hline \multicolumn{3}{|l|}{ Escolaridad } \\
\hline Ninguna & 6 & 6.6 \\
\hline Primaria & 22 & 24.2 \\
\hline Secundaria & 29 & 31.9 \\
\hline Preparatoria & 25 & 27.5 \\
\hline Universidad & 9 & 9.9 \\
\hline \multicolumn{3}{|l|}{ Estado civil } \\
\hline Casado & 33 & 36.3 \\
\hline Soltero & 16 & 17.6 \\
\hline Unión libre & 29 & 31.9 \\
\hline Viudo & 13 & 14.3 \\
\hline \multicolumn{3}{|l|}{ Hospital } \\
\hline IMSS & 26 & 28.6 \\
\hline ISSSTE & 22 & 24.2 \\
\hline Hospital General & 43 & 47.3 \\
\hline \multicolumn{3}{|l|}{ Ansiedad } \\
\hline Normal & 19 & 20.9 \\
\hline Probable ansiedad & 25 & 27.5 \\
\hline Ansiedad & 47 & 51.6 \\
\hline \multicolumn{3}{|l|}{ Depresión } \\
\hline Normal & 47 & 51.6 \\
\hline Probable depresión & 21 & 23.1 \\
\hline Depresión & 23 & 25.3 \\
\hline
\end{tabular}

pacientes es similar en relación con el grado de escolaridad $(p=.32)$ y estado civil $(p=.74)$; sin embargo, al comparar el lugar donde recibe la atención, se identificó que la ansiedad se presenta con diferente intensidad en cada unidad de atención ( $p=.05$; Tabla 2).

Se encontró que la ansiedad no es diferente en los pacientes que trabajan, en comparación con aquellos que no ( $p=.08$ ). Se observó que la ansiedad es similar en los que conocen su etapa clínica en comparación con aquellos que no la conocen ( $p=789$; Tabla 3).

Otro hallazgo fue que la depresión en las pacientes con cáncer es similar en los diferentes grados de escolaridad de las pacientes $(p=.323$ ). La depresión es diferente de acuerdo con la unidad de atención $(p=.001)$, los pacientes que son atendidos en el hospital resultaron con menor depresión en comparación con los que son atendidos en las otras instituciones (Tabla 4).

La depresión se presentó de manera similar en las pacientes que trabajan, en comparación con aquellas que no $(p=.095)$, lo mismo que en los que conocen su etapa clínica en comparación con aquellos que no la conocen ( $p=.231$; Tabla 5).

Actualidades en Psicología, 34(129), 2020, 109-118 
Tabla 2

Diferencias en ansiedad por escolaridad, estado civil y unidad de atención

\begin{tabular}{|c|c|c|c|c|c|}
\hline Ansiedad & M & $D T$ & $M d n$ & $H$ & $p$ \\
\hline \multicolumn{6}{|l|}{ Escolaridad } \\
\hline Ninguna & 10.17 & 2.22 & 11.00 & \multirow{5}{*}{4.671} & \multirow{5}{*}{.32} \\
\hline Primaria & 11.32 & 3.79 & 12.00 & & \\
\hline Secundaria & 9.72 & 3.01 & 10.00 & & \\
\hline Preparatoria & 9.68 & 3.31 & 10.00 & & \\
\hline Universidad & 10.44 & 2.55 & 11.00 & & \\
\hline \multicolumn{6}{|l|}{ Estado civil } \\
\hline Casado & 9.94 & 2.97 & 11.00 & \multirow{4}{*}{1.216} & \multirow{4}{*}{.74} \\
\hline Soltero & 9.69 & 3.53 & 9.00 & & \\
\hline Unión libre & 10.66 & 3.55 & 11.00 & & \\
\hline Viudo & 10.46 & 2.87 & 11.00 & & \\
\hline \multicolumn{6}{|l|}{ Unidad de atención } \\
\hline IMSS & 9.54 & 2.17 & 10.00 & \multirow{3}{*}{5.792} & \multirow{3}{*}{.05} \\
\hline ISSSTE & 9.55 & 2.11 & 10.00 & & \\
\hline Hospital General & 10.93 & 4.03 & 12.00 & & \\
\hline
\end{tabular}

Nota. $H$ = prueba $\mathrm{H}$ de Kruskal Wallis

Tabla 3

Diferencias en ansiedad en los que trabajan y no, y en los que conocen y no la etapa clínica de su enfermedad

\begin{tabular}{lccccc}
\hline Ansiedad & $M$ & DT & Mdn & $U$ & $p$ \\
\hline Trabaja & & & & & \\
Si & 9.54 & 2.87 & 10.00 & 798.000 & .081 \\
No & 10.69 & 3.41 & 11.00 & & \\
Conoce la etapa clínica & & & & & \\
Si & 10.19 & 3.29 & 11.00 & & \\
No & 10.20 & 3.20 & 10.00 & 995.500 & .789 \\
\hline
\end{tabular}

Nota. $U$ = prueba $U$ de Mann Whitney 
Tabla 4

Diferencias en depresión por escolaridad, estado civil y unidad de atención

\begin{tabular}{|c|c|c|c|c|c|}
\hline Depresión & M & $D T$ & Mdn & $H$ & $p$ \\
\hline \multicolumn{6}{|l|}{ Escolaridad } \\
\hline Ninguna & 7.83 & 3.86 & 7.50 & \multirow{5}{*}{.919} & \multirow{5}{*}{.922} \\
\hline Primaria & 6.82 & 4.99 & 6.50 & & \\
\hline Secundaria & 7.79 & 4.64 & 8.00 & & \\
\hline Preparatoria & 7.56 & 4.95 & 8.00 & & \\
\hline Universidad & 7.44 & 4.27 & 8.00 & & \\
\hline \multicolumn{6}{|l|}{ Estado civil } \\
\hline Casado & 7.39 & 5.27 & 7.00 & \multirow{4}{*}{1.746} & \multirow{4}{*}{.627} \\
\hline Soltero & 6.50 & 4.78 & 5.50 & & \\
\hline Unión libre & 7.52 & 3.98 & 8.00 & & \\
\hline Viudo & 8.69 & 4.44 & 8.00 & & \\
\hline \multicolumn{6}{|l|}{ Unidad de atención } \\
\hline IMSS & 9.58 & 4.46 & 9.50 & \multirow{3}{*}{14.042} & \multirow{3}{*}{.001} \\
\hline ISSSTE & 8.55 & 3.93 & 8.50 & & \\
\hline Hospital General & 5.63 & 4.47 & 6.00 & & \\
\hline
\end{tabular}

Nota. $\mathrm{H}=$ prueba $\mathrm{H}$ de Kruskal Wallis

Tabla 5

Diferencias en depresión en los que trabajan y no, y en los que conocen y no la etapa clínica de su enfermedad

\begin{tabular}{lccccc}
\hline \multicolumn{1}{c}{ Depresión } & $M$ & DT & Mdn & $U$ & $p$ \\
\hline Trabaja & & & & & \\
Sí & 8.36 & 4.54 & 8.00 & & \\
No & 6.79 & 4.66 & 6.50 & 806.500 & \\
Conoce la etapa clínica & & & & & \\
Sí & 6.88 & 4.89 & 6.50 & & \\
No & 7.96 & 4.43 & 8.00 & 879.000 & .231 \\
\hline
\end{tabular}

Nota. $U$ = prueba $U$ de Mann Whitney

\section{Discusión}

En este estudio se encontró que la mitad de los sujetos resultaron con ansiedad, este hallazgo se acerca a lo encontrado por Nikbakhsh et al. (2014), sin embargo, es superior a lo reportado por Watts et al. (2015) e inferior al resultado de Valderrama y Sánchez (2017). Las diferencias pueden estar relacionadas a que el trabajo de Watts et al. (2015) parte de una revisión sistemática de la literatura donde incluyeron artículos de pacientes con cáncer de ovario y los resultados del análisis de la ansiedad son de antes, durante y después del tratamiento. Mientras que en el presente estudio, la población fue de mujeres con cáncer de mama, y la medición se hizo durante el tratamiento sin considerar el tiempo que la paciente tenía en este.

Por otra parte, las diferencias con el estudio de Valderrama y Sánchez (2017) pueden explicarse en que en su estudio incluyeron pacientes con cáncer de mama en estadío avanzado o diseminado, mientras en este estudio no se analizó el estadío de la 
enfermedad. Los resultados obtenidos de ansiedad y depresión confirman lo establecido por Watson et al. (1988), Folkman \& Lazarus (1984) y Almanza-Muñoz y Holland (2000), quienes indican que la enfermedad oncológica se presenta con respuestas conductuales en los pacientes, estas incluyen la ansiedad y la depresión.

En este estudio, una cuarta parte de la población presentó depresión, similar a lo reportado anteriormente por otros autores (Nikbakhsh et al., 2014; Valderrama \& Sánchez, 2017; Watts et al., 2015); sin embargo, es inferior a lo encontrado por Fuchs-Taralovsky et al. (2013). La discrepancia pudiera explicarse en que la población de dicho estudio fue de mujeres hospitalizadas, a diferencia de este; en el cual las mujeres acudían a los diferentes hospitales a recibir el tratamiento. Este dato indica que el contexto de la paciente es de gran importancia, por lo que se sugiere realizar estudios donde se identifique si la presencia de sintomatología depresiva es diferente en pacientes hospitalizados en comparación con los que se encuentran en su contexto familia; ya que la literatura indica que, ante estos problemas, la familia puede ser la principal fuente de apoyo, así como fungir como factor protector (Fuchs-Taralovsky et al., 2013).

Aunque los resultados sobre la ansiedad y depresión obtenidos en el presente estudio no permiten explicar la presencia de la sintomatología ansiosa o depresiva, resultan preocupantes y constituyen un importante acercamiento a esta problemática; ya que en muchas ocasiones durante el tratamiento del cáncer poco se toma en cuenta el estado psicoemocional de los pacientes. Por tanto, se considera substancial que los trabajadores de la salud dispongan de herramientas para la identificación de estos problemas; así como plantear e implementar intervenciones para mejorar el estado de ánimo de las pacientes y de ser necesario, canalizarlos para recibir atención especializada.

Algunos autores indican que la ansiedad es una respuesta ante la percepción y evaluación subjetiva de una situación o acontecimiento amenazante. En este sentido, el cáncer es un problema de salud que amenaza la vida, donde algunas de las situaciones que pudieran generar ansiedad en las pacientes con cáncer es el bajo conocimiento sobre la enfermedad y el tratamiento, lo que conlleva incertidumbre. Sin embargo, estas variables no han sido abordadas en esta población, por lo que se considera importante incluirlas en futuros estudios (Sandín \& Chort, 1995).

Por otra parte, los estudios identificados sobre la ansiedad y depresión poseen un diseño descriptivo; en este estudio se buscó conocer las diferencias en la ansiedad y depresión por variables contextuales. Pese a que Pintado-Cucarella (2016) plantea que el cáncer conlleva un desequilibrio en el bienestar físico, emocional y contextual por no tener la misma funcionalidad; en este estudio no se encontró relación estadísticamente significativa entre la ansiedad y depresión con la edad de las pacientes con cáncer. Se observó que la ansiedad y depresión no difieren de acuerdo con el nivel escolar, estado civil, condición laboral y conocimiento sobre la etapa clínica de la enfermedad.

Los resultados en cuanto a la escolaridad son diferentes a lo encontrado por Berrospi-Reyna et al. (2017) quienes reportaron que la depresión es diferente de acuerdo con el nivel de escolaridad. Además, lo observado en esta investigación concuerda con lo indicado por Lerma-Talamantes et al. (2017) al confirmar que la depresión y ansiedad es similar de acuerdo con el empleo y estado civil de la paciente. De tal manera que, ante la presencia de ansiedad y depresión en las mujeres con cáncer de mama, pueden estar presentes otras variables mediadoras como el tipo de empleo, tiempo de convivencia con la familia, apoyo social, apoyo familiar, ayuda gubernamental, entre otras que no fueron consideradas en esta investigación. 
Por último, se encontraron diferencias estadísticamente significativas en la depresión o ansiedad por institución en que recibe el tratamiento, es decir, la depresión en las mujeres que son atendidas en el Hospital General fue menor en comparación con las que son atendidas en el IMSS o ISSSTE. Mientras que la ansiedad fue menor en las mujeres que son atendidas en el IMSS en comparación con las que son atendidas en el Hospital General o ISSSTE.

Las diferencias encontradas en la depresión y ansiedad por institución de atención, explican la importancia que tiene el contexto en que la paciente con cáncer es atendida, donde la institución propiamente puede no ser el factor directo; pues se piensa que pueden estar implicadas otras variables como la empatía con el proveedor del tratamiento, el tiempo de haber recibido el diagnóstico y el apoyo familiar al recibir el diagnóstico y durante el tratamiento. En este sentido, se sugiere continuar sobre esta línea de investigación y en futuros estudios integrar las variables ya mencionadas.

Se concluye que es importante cuidar el estado emocional de las pacientes con cáncer, ya que en el presente estudio se encontró que la mitad de las pacientes resultó con ansiedad y una cuarta parte con depresión. No se observó relación entre la ansiedad y depresión con la edad de las pacientes con cáncer. No se encontraron diferencias estadísticamente significativas en la ansiedad y depresión de acuerdo con el nivel escolar, estado civil, ocupación laboral y conocimiento sobre la etapa clínica de la enfermedad; únicamente se encontraron diferencias estadísticamente significativas en la depresión o ansiedad por institución en que recibe el tratamiento.

Se considera que una limitante para la generalización de los resultados es el muestreo de tipo no probabilístico; por lo tanto, se recomienda tomar con cautela estos resultados y continuar realizando estudios para clarificar la asociación entre la ansiedad y depresión con las variables ya mencionadas, así como otras posibles variables mediadoras que puedan confundir la interpretación de los resultados, por ejemplo, el funcionamiento familiar, la espiritualidad y religiosidad y el apoyo social.

\section{Referencias}

Almanza-Muñóz, J. J., \& Holland, J. C. (2000). Psico-oncología: estado actual y perspectivas futuras. Revista del instituto nacional de cancerología, 46(3), 196-206.

Berrospi-Reyna, S., Herencia-Souza, M., \& Soto, A. (2017). Prevalencia y factores asociados a la sintomatología depresiva en mujeres con cáncer de mama en un hospital público de Lima, Perú. Acta Med Peru, 34(2), 95-100.

Folkman, S., \& Lazarus, R. S. (1984). If it changes, it must be a process: A study of emotion and coping during three stages of a collage examination. J Pers Soc Psychol, 50, 992-1033. http://dx.doi.org/10.1037/00223514.48.1.150

Fuchs-Tarlovsky, V., Bejarano, M., Álvarez, K., Godoy, M., \& Fernández, N. C. (2013). Efecto de la presencia de los familiares sobre la depresión en mujeres hospitalizadas con Cáncer. Revista Venezolana de Oncología, 25(3), 190-195.

Galindo, O., Benjet, C., Juárez, F., Rojas, E., Riveros, A., Aguilar, J. L. Álvarez, M. A., \& Alvarado, S. (2015). Propiedades psicométricas de la Escala Hospitalaria de Ansiedad y Depresión (HADS) en una población de pacientes oncológicos mexicanos. Salud Mental, 38(4), 253-58.

Gómez, O., Sesma, S., Becerril, V. M., Knaul, F. M., Arreola, H., \& Frenk, J. (2011). Sistema de Salud de México. Salud Pública de México, 53(2), S220-\$232. 
Lerma-Talamantes, A., Larios-Jiménez, F. E., Daneri-Navarro, A., Lerma, C., \& RoblesGarcía, R. (2017). Factores asociados con síntomas de depresión y ansiedad en pacientes mexicanas con cáncer de mama. Revista Latinoamericana de Revista Conductual, 8(1), 16-23. http://www.revistas.unam.mx/index.php/rlmc/article/ view/65125/57131

Nikbakhsh, N. Moudi, S., Abbasian, S., \& Khafri, S. (2014). Prevalence of depression and anxiety among cancer patients. Caspian J Intern Med, 5(3), 167-170.

Organización Mundial de la Salud (2012). Programas nacionales de lucha contra el Cáncer. Directrices sobre política y gestión. http://www.who.int/cancer/media/en/423.pdf

Organización Mundial de la Salud (2018a). Cáncer. https://www.who.int/es/news-room/ fact-sheets/detail/cancer

Organización Mundial de la Salud (2018b). Depresión. https://www.who.int/es/newsroom/fact-sheets/detail/depression

Pintado-Cucarella, S. (2016). Alteraciones de la autoestima y la imagen corporal y síntomas de depresión y ansiedad en una muestra de pacientes mexicanas con cáncer de mama. Salud pública de México, 58(4), 400.

Sandín, B., \& Chorot, P. (1995). Concepto y categorización de los trastornos de ansiedad. En A. Belloch, B. Sandín y F. Ramos (Eds.), Manual de Psicopatología, Vol. 2 (pp. 53-80). McGraw-Hill.

Sarason, I. G. \& Sarason B. R. (2006). Psicopatología. Psicología anormal: el problema de la conducta inadaptada $\left(11^{\circ}\right.$ ed.). Pearson Educación.

Valderrama, M. C. \& Sánchez, R. (2017). Trastornos de ansiedad y depresión en relación con la calidad de vida de pacientes con cáncer de mama en estadio localmente avanzado o diseminado. Revista Colombiana de Psiquiatría, 47(4), 211-220. https://doi.org/10.1016/j.rcp.2017.04.003

Watson, M., Greer, S., Young, J., Inayat, Q., Burgess, C., \& Robertson, B. (1988). Development of a questionnaire measure of adjustment to cancer: The MAC scale. Psychol Med, 18, 203-9. http://dx.doi.org/10.1017/S0033291700002026

Watts, S., Prescott, P., Mason, J., McLeod, N., \& Lewith, G. (2015). Depression and anxiety in ovarian cancer: a systematic review and meta-analysis of prevalence rates. $B M J$ open, 5(11), e007618.

Zigmond, A. S., \& Snaith, R. P. (1983). The hospital Anxiety and depression scale. Acta Psychiatr Scand, 67(6), 361-370. 